\title{
Cloud Computing: Environment of Next Generation Technology
}

\author{
Rajan Kumar Yadav ${ }^{1}$, Avinash Thakur ${ }^{2}$, Manoranjan Kumar ${ }^{3}$ \\ ${ }^{1,2,3}$ (Department of Computer, JNTU Hyderabad, India)
}

\begin{abstract}
Cloud computing is the convergence of virtualization, Distributed Applications, Grid, Maturity of enterprise software application and enterprise IT Management. It is a tremendously attractive technology as it enables a fundamental shift from capital intensive focus to a flexible operational management model. Basically it is characterized by on demand computing, based on pay per use pricing model. This technology enables the user to use the system without the need to know the physical location and configuration of the system that delivers the services. At the same time it also offers participation of less manpower and zero maintenance of the services. This paper is based on the description of cloud computing technology and its numerous benefits. Furthermore this paper also highlights the Environment of Cloud Computing and Consideration on Cloud Adoption.
\end{abstract}

Keywords: Cloud Infrastructure, Grid Computing, Information Communication Technology (ICT), Infrastructure as a Service (IaaS), Platform as a Service (PaaS), Software as a Service (SaaS), Virtualization

\section{Introduction}

Each day witnesses the growing popularity of the Internet and the web along with the availability of powerful hand-held computing mobile and sensing devices. The Internet is changing the way we interact, manage our lives, conduct business and access or deliver services. As it is the rule of technology that "technology supports technology" and as the time passes, technology always needs higher form of technological services. As we know it that entire computing paradigm has shifted from computer desktop to mobile devices. In this scenario cloud computing is expected to be one of the fastest growing technologies in the upcoming years. It is a technological advancement which focuses on the way in which we design computing system, develop application and leverage existing services for building software.

Cloud computing is based on the concept of dynamic provisioning which is applied not only to services but also to compute capability, storage, networking and information technology(IT) infrastructure in general. It allows renting infrastructure, run time environments and service on pay per use basis. Today any one with the help of Internet connection can subscribe to cloud services, grow and shrink the infrastructure, serve its application according to the demand and pay only for the time these resources has been used. Cloud computing is the convergence of virtualization, distributed application, grid, maturity of enterprise software applications and IT management.

In the era of recession, due to debt rising and inflation there is a need of cost contraction rather than cost expansion. There is an even stronger need for organizations to improve efficiency by using collaborative solution in real time exchange of information. To fulfill this overall objective cloud computing is the evolving technology for the next generation, which is going to create a difference in the overall scenario of information communication technology (ICT) [1], [2].

\section{Cloud Architecture}

According to the cloud security alliance and NIST guidelines [2], cloud computing has four different deployment model namely private, community, public and hybrid.

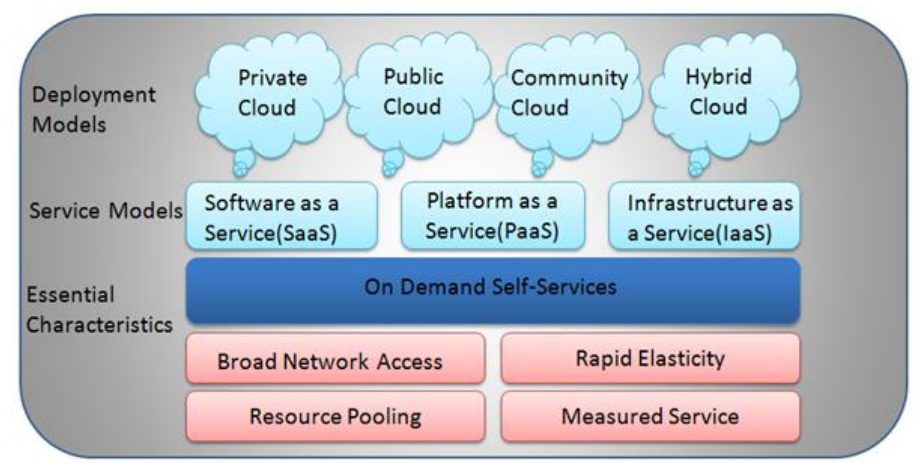

Fig. 2.1 Cloud Computing Framework [NIST] 


\subsection{Service Models for the Cloud}

\subsubsection{Infrastructure as a Service(IaaS)}

The provisioning of processing time, storage, networks, and other fundamental computing Resources, made available by Cloud service providers for customer's use is known as Infrastructure as a Service (IaaS). These resources can be used to deploy and run arbitrary software which can include operating systems and applications. The service provider is responsible for the management of the underlying Cloud infrastructure [3], [4]. Examples include: Amazon EC2, Zenith's Proud, etc.

\subsubsection{Platform as a Service (PaaS)}

The capability provided by Cloud service providers for customers to develop and host applications is known as Platform as a Service (PaaS). The customer does not manage or control the underlying infrastructure that includes network, servers, operating systems, software for application development and these are managed by the service provider. Examples include: SalesForce.com'sForce.com, Wolf Framework's Wolf PaaS, Windows Azure, etc.

\subsubsection{Software as a Service (SaaS)}

The delivery of software applications over the internet while being managed by the service provider, is known as Software as a Service (SaaS). Customers are not concerned with the regular maintenance, up gradation and the management of the underlying computing infrastructure that is used to host the applications. The applications are accessible from various client devices through web browsers. Examples include: Gmail, Facebook, SalesForce.com, Zoho CRM, etc.

\subsection{Deployment Models for the Cloud}

\subsubsection{Private Cloud}

Private Cloud is the Cloud which operates dedicatedly for a single organization. Private cloud infrastructure may be set-up On-Premise or off-premise and may be managed either internally or by third-party service providers. Private clouds offer highest level of security and control but are expensive. An example would be the NASA's Nebula Private Cloud which is an infrastructure-as-a-service implementation for scientific data and Web-based applications.

\subsubsection{Public Cloud}

Public Cloud is the Cloud in which services are available to the general public or a large group of companies with whom resources are shared. It is owned by a third-party selling Cloud services. An example would be the Amazon EC2 Cloud wherein the service provider, in this case Amazon, rents out storage space to businesses, governments, and individuals alike.

\subsubsection{Community Cloud}

Community Cloud is the Cloud whose infrastructure is shared by several organizations and supports a specific community that has shared concerns. The community Cloud may be deployed On-Premise or OffPremise and may be managed by organizations collectively or by a third-party Cloud services provider. An example would be the Community Cloud being built by the Mount Sinai Hospital in Toronto, Canada that will give 14 area hospitals shared access to fetal ultrasound application and Data storage for patient information.

\subsubsection{Hybrid Cloud}

Hybrid Cloud is the Cloud which uses a combination of two or more clouds (public, private or community). The Clouds could be individually managed by multiple Cloud service providers but are bound together by proprietary technology to enable data and application portability. E.g. a company may use a public cloud for storing archived data but may use a private cloud for storing critical information like customer details and information.

\section{Environment of Cloud Computing}

It is an environment in which no long term commitments are required. System can be used as and when required. The emergence of the "as a service paradigm has made the delivery of IT services a utility". At the same time it would be right to say that the cloud would rapidly change the way it is supplied and consumed [7], [8]. It has become popular buzz word and has been widely used to refer different technological services and concept. 


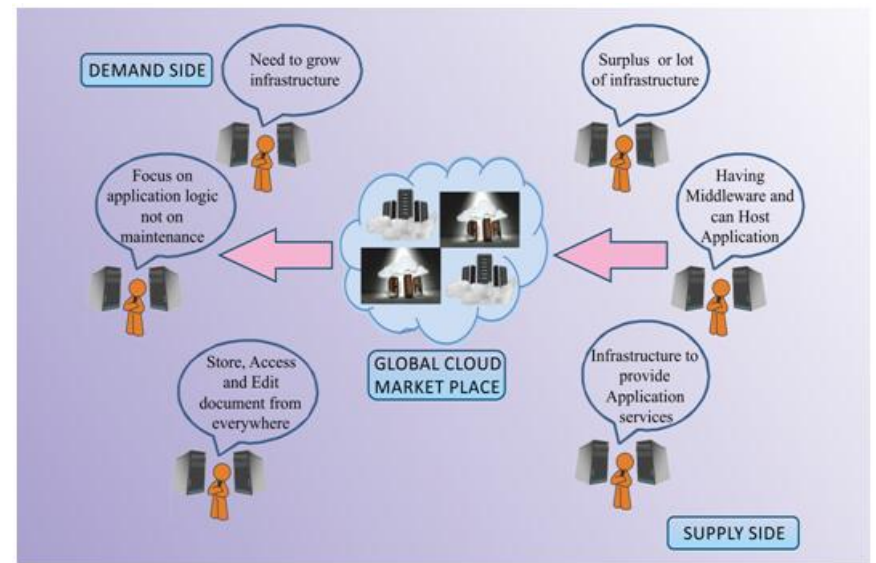

Fig. 3.1 Cloud Environment: Demand and Supply Scenario

A macroscopic view of the cloud environment signifies two sides, i.e. Demand Side and Supply side. Both are mutually dependent and synchronized. One can have a closer view on the demand side and the supply side as follows.

\subsection{Demand Side}

$>$ One wants to grow infrastructure but do not know how long.

$>$ One cannot invest on infrastructure in the early phase of business.

$>$ One wants to focus on application logic and not on maintenance and scalability issue.

\subsection{Supply Side}

$>$ One has lot of infrastructure, which can be rent out.

$>$ One has surplus of infrastructure, which can be used off.

$>$ One has infrastructure, middleware and host application.

On summarizing demand side and supply side, the cloud environment provides following benefits:

- Device and storage can be availed from everywhere.

- Small enterprises can start up and attempt to translate their ideas into business result more quickly without excessive upfront cost.

- System developer can concentrate on the business logic rather than dealing with the complexity of infrastructure management and scalability.

- Large enterprises can offload some of their activity to cloud based system.

\subsection{Expectations from Cloud Based Solutions}

\section{Considerations on Cloud Adoption}

> Automated Service provision, monitoring and management for achieving Agility - enabling service providers to focus on managing hardware, software, services as well as end usage outcome, rather than for businesses to set up extensive and expensive operations organizations.

> Internet based Access model using high capacity bandwidth and ubiquitous connectivity enabling businesses to deliver business applications to their customers and associates on multiple devices with round-the-clock.

> Dynamic and flexible Technology model to align with the changing needs of the business enabling businesses to provision technology resources based on demand and usage at the right level and at the right time, rather than having to plan upfront for capacity.

$>$ Rapid Innovation in services, features and operating models leveraging the capabilities of service provider(s) - as compared to the need for internal and isolated investments and enabling the full impact of Innovation to reach all consumers rapidly.

$>$ Usage based Business model that enables businesses to spend based on consumption - allowing businesses to provide their technology investments with full alignment to business needs at a granular level.

All of the above expectations have evolved over the years as the scale and complexity of the portfolio has grown. There have been successful adoption of technological innovations like Grid computing, 
Virtualization and SOA, which have delivered some of these expectations; Cloud solutions are seen as the grand convergence of all these innovations to deliver holistic, outcome based business capabilities [10].

1.6. Challenges and Risks with Cloud Based Solutions

> Scalability and Performance of the service provided, especially the ability to support volume / transaction / user heavy requirements without diluting the user / business experience and outcomes.

$>$ Complexity of Governance, Audit \& Compliance associated with service models as compared with the relatively easy model of Buy / License and use that is prevalent today.

$>$ Legal aspects related to data, visibility, and access and information management; especially in the BFS industry that is heavily regulated globally.

$>$ Insufficient proof of success of the Service Providers - enabling businesses to outsource full spectrum of service capabilities more effectively, efficiently and to manage scale in an elastic manner.

$>$ Increased probability of risk \& exposure to potential issues related to business operations, confidentiality and compliance, which are critical in the financial service industry.

1.7. Aspects Accelerating Cloud based Solutions

> Success of outsourcing and the comfort within the organization, at all levels, with entrusting significant parts of the business and technology operations with services from multiple service providers; more than $70 \%$ of financial institutions have significant success and measurable results of $16-18 \%$ savings on their investments.

$>$ Intent to achieve value with next generation technology \& services model evolving from the current model where almost all opportunities of value generation have been fully leveraged.

> Possibilities of offering Financial Services on Cloud platforms to other peer institutions based on the robustness, coverage, capacity and maturity.

> Promise of shifting the IT Spend pattern from a BAU heavy model to a capability generating model; influenced significantly by the decrease in the budgets at a time when business needs are rapidly growing.

Promise of business agility with the ability to scale and respond to business variations as needed rather than building redundant capacity; research indicates that in excess of 30\% of computing power and $15 \%$ of human capacity is typically unused in most of the financial institutions, during normal business.

\section{Conclusion}

The long-term vision of cloud computing is to fully realize the utility model that drives it service offering. It is envisioned that new technological developments and the increased familiarity with cloud computing delivery model, will lead to establishment of a global market for trading and computing utilities. It is expected to be one of the fastest growing technologies in the coming years, as it has already brought economic, environmental and technological benefits. As we are leaping towards a new decade, the cloud could become the nest revolutionary wave. The scalability and flexible nature of the cloud holds promise to organization to not just alter the way they manage IT but also transform business. In times to come we could see the cloud as the Gen Next Technology.

\section{Reference}

[1]. Mihai Tudor, ICT Cloud Computing, Cisco Expo, Bucharest, April 2013

[2]. Fang Liu, Jin Tong, Jian Mao, Robert Bohn, John Messina, Lee Badger and Dawn Leaf, NIST Cloud Computing Reference Architecture, NIST Special Publication 500-292, NIST SP 500-292

[3]. Zoran Pantić and Muhammad Ali Babar, Guidelines for Building a private Cloud Infrastructure, Tech Report TR-2012-153, ISBN: 978-87-7949-254-7,IT University of Copenhagen, Denmark, 2012

[4]. Penny Pritzker, NIST Cloud Computing Standards Roadmap, NIST Special Publication 500-291, Version 2, July 2013

[5]. Nelson Gonzalez, Charles Miers, Fernando Red'igolo, Marcos Simpl'icio, Tereza Carvalho, Mats N*aslund and Makan Pourzandi, A quantitative analysis of current security concerns and solutions for cloud computing, Gonzalez et al. Journal of Cloud Computing: Advances, Systems and Applications 2012, 1:11

[6]. Introduction to Cloud computing, Dialogic Corporation, 07/10, 12023-01

[7]. Qi Zhang, Lu Cheng, Raouf Boutaba, Cloud computing: state-of-the-art and research challenges, J Internet Serv Appl (2010) 1: 7 18 DOI 10.1007/s13174-010-0007-6

[8]. Keith Jeffery, Burkhard Neidecker, The future of cloud computing, Public Version 1.0

[9]. Gerard Conway and Edward Curry, Managing cloud computing: A life cycle Approach, Value Institute's Cloud Computing working group, Grant CC/2009/0801

[10]. Lutz Schubert, Keith Jeffery, Research in Future Cloud Computing, Expert Group Report, Public Version 1.0

[11]. Wayne and Timothy, Guidelines on Security and Privacy in Public Cloud Computing,NIST Publication 800-144, Jan.2011 


\section{Author's Profile}

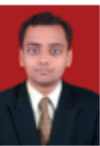

Rajan Kumar Yadav. He is an IBM DB2 Academic Associate. He has received B.E. degree from MIT Pune, India. $\mathrm{He}$ is pursuing $\mathrm{M}$. Tech in Computer Science and Engineering from Jawaharlal Nehru Technological University (JNTU), Hyderabad, India. His area of Interest is Cloud Computing, Programming Languages and Database. He has done various research works in his area of research to contribute to IT industry and in the field of Open Source Technology.

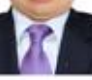

Avinash Thakur. He has received B.E. degree from MIT Pune, India. He is pursuing M. Tech in Computer Science and Engineering from Jawaharlal Nehru Technological University (JNTU), Hyderabad, India. His area of interest is Computer Network, Digital Signal Processing, Mobile Computing, Theory of Computation, Cloud Computing and Engineering Mechanics. He is having 5-years of teaching experience in the field of Engineering Mechanics.

Manoranjan Kumar. He has received B.E. degree from MIT Pune, India. He is pursuing M. Tech in Computer Science and Engineering from Jawaharlal Nehru Technological University (JNTU), Hyderabad, India. His area of interest is Data Structure, Compiler Design, Theory of Computation, Digital Signal Processing and Programming Languages. 